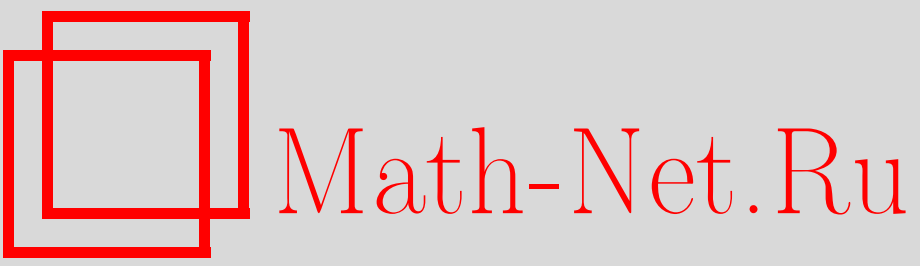

Г. В. Кузнецов, Геометрия общего движения крови в системе кровообращения, Итоги науки и техн. Сер. Соврем. мат. и ее прил. Темат. обз., 2020, том $182,51-54$

DOI: https://doi.org/10.36535/0233-6723-2020-182-51-54

Использование Общероссийского математического портала Math-Net.Ru подразумевает, что вы прочитали и согласны с пользовательским соглашением

http://www. mathnet.ru/rus/agreement

Параметры загрузки:

IP: 54.174 .149 .18

26 апреля 2023 г., 07:25:44 


\title{
ГЕОМЕТРИЯ ОБЩЕГО ДВИЖЕНИЯ КРОВИ В СИСТЕМЕ КРОВООБРАЩЕНИЯ
}

\author{
(C) 2020 г. $\quad$ Г. В. КУЗНЕЦОВ
}

\begin{abstract}
АннотАция. Работа посвящена изучению геометрических характеристик движения крови в системе кровообращения. Рассмотрен случай, когда геометрия движения частиц крови моделируется как геометрия движения в субпроективном пространстве, отнесенном к неголономным реперам.
\end{abstract}

Ключевъе слова: турбулентное движение, неголономный репер, дифференциальная форма.

\section{GEOMETRY OF THE GENERAL MOTION OF BLOOD IN THE CIRCULATORY SYSTEM}

\author{
(c) 2020 G. V. KUZNETSOV
}

\begin{abstract}
This work is devoted to the study of geometric characteristics of the motion of blood in the circulatory system. We consider the case where the geometry of motion of blood particles is modeled as the geometry of motion in the subprojective space referred to a nonholonomic frame.
\end{abstract}

Keywords and phrases: turbulent motion, nonholonomic frame, differential form.

AMS Subject Classification: 53C12, 57R 15

Предположим, что кровь движется турбулентно, и из одной точки сосуда в другую частица крови смещается по некоторому пути. Рассмотрим случай, при котором не существует поверхностей, на которых бы располагались линии тока и вихревые линии. Поэтому рассмотрение геометрии такого движения частиц крови удобнее проводить как геометрию движения в субпроективном пространстве, отнесенном к неголономным реперам.

В касательном пространстве к трехмерному субпроективному пространству зададим репер, определяемый точкой $x \in C^{3}$ и векторами первого и второго порядка. Уравнения перемещения такого репера имеют вид:

$$
d \boldsymbol{x}=\omega^{A} \boldsymbol{e}_{A}, \quad d \boldsymbol{e}_{A}=\omega_{A}^{B} \boldsymbol{e}_{B}+\omega^{B} \boldsymbol{e}_{A B},
$$

где $\boldsymbol{e}_{A B}$ - векторы, образующие совместно с векторами первого порядка репер второго порядка, а также

$$
e_{A B} \neq e_{B A}
$$

Формы $\omega^{1}, \omega^{2}, \omega^{3}$ являются линейно-независимыми, $\omega^{1} \vee \omega^{2} \vee \omega^{3} \neq 0, \omega^{1} \wedge \omega^{2} \wedge \omega^{3} \neq 0$ и являются структурными параметрами сердечно-сосудистой системы. Дифференциальные формы $\omega^{A}$ и $\omega_{B}^{A}$ из уравнений (1) удовлетворяют уравнениям структуры субпроективного пространства:

$$
D \omega^{A}=\omega^{B} \wedge \omega_{B}^{A}, \quad D \omega_{B}^{A}=\omega_{B}^{K} \wedge \omega_{K}^{A}+R_{B K L}^{A} \omega^{K} \wedge \omega^{L},
$$

где $R_{B K L}^{A}$ - тензор кривизны субпроективного пространства. В качестве структурной группы этого пространства возьмем ортогональную группу $O(3)$, инвариантные формы которой удовлетворяют уравнениям $\sigma_{A}^{B}+\sigma_{B}^{A}=0$, где $\omega_{B}^{A}\left(\omega^{A}=0\right)=\sigma_{B}^{A}$. Тогда формы $\omega_{B}^{A}$ также удовлетворяют 
уравнениям:

$$
\omega_{B}^{A}+\omega_{A}^{B}=0, \quad \omega_{A}^{A}=0 .
$$

Найдем выражение для градиента функции $\phi$, дивергенцию и ротора для рассматриваемого субпроективного пространства. Выражение для градиента функции, как легко видно, будет иметь точно такой же вид, как и в случае евклидова пространства, так и для субпроективного пространства, отнесенного к голономному реперу:

$$
\operatorname{grad} \phi=\frac{e^{1} d \phi \wedge \omega^{2} \wedge \omega^{3}+e^{2} d \phi \wedge \omega^{3} \wedge \omega^{1}+e^{3} d \phi \wedge \omega^{1} \wedge \omega^{2}}{\omega^{1} \wedge \omega^{2} \wedge \omega^{3}}
$$

где $e^{1}, e^{2}, e^{3}$ - взаимные векторы к векторам данного репера. Пусть $\boldsymbol{v}$ - вектор скорости частицы крови, который представим в виде $\boldsymbol{v}=v^{A} \boldsymbol{e}_{A}$. Дифференцируя это равенство и используя второе равенство из (1), получим

$$
d \boldsymbol{v}=\left(d v^{A}+v^{B} \omega_{B}^{A}\right) \boldsymbol{e}_{A}+v^{A} \omega^{B} \boldsymbol{e}_{A B} .
$$

После преобразований в правой части, получаем:

$$
\begin{aligned}
\omega^{1} \wedge \omega^{2} \wedge \omega^{3} \operatorname{div} \boldsymbol{v}=\left(d v^{1}+v^{B} \omega_{B}^{1}\right) \wedge \omega^{2} \wedge \omega^{3}+\left(d v^{2}+v^{B} \omega_{B}^{2}\right) \wedge \omega^{3} \wedge \omega^{1}+ \\
+\left(d v^{3}+v^{B} \omega_{B}^{3}\right) \wedge \omega^{1} \wedge \omega^{2}+\left(v^{K} a_{K A}^{A}\right) \omega^{1} \wedge \omega^{2} \wedge \omega^{3},
\end{aligned}
$$

где в последнем слагаемом предполагается вначале сумма по $A$, а затем сумма по $K \neq A$. Для нахождения выражения для ротора вектора скорости крови воспользуемся формулой

$$
\begin{aligned}
& -\operatorname{rot} \boldsymbol{v} d \tau=e_{1}\left(\omega^{1} \wedge \omega^{2} \wedge\left(d v^{2}+v^{L} \omega_{L}^{2}\right)+\omega^{1} \wedge \omega^{3} \wedge\left(d v^{3}+v^{L} \omega_{L}^{3}\right)+d \tau\left(v^{K} a_{K 3}^{2}-v^{K} a_{K \neq K}^{3}\right)\right)+ \\
& +\boldsymbol{e}_{2}\left(\omega^{2} \wedge \omega^{1} \wedge\left(d v^{1}+v^{L} \omega_{L}^{1}\right)+\omega^{2} \wedge \omega^{3} \wedge\left(d v^{3}+v^{L} \omega_{L}^{3}\right)+d \tau\left(v^{K} a_{K 1}^{3}-v^{K} a_{K 3}^{1} \begin{array}{c}
1 \neq K \\
3 \neq K
\end{array}\right)\right)+ \\
& +\boldsymbol{e}_{3}\left(\omega^{3} \wedge \omega^{1} \wedge\left(d v^{1}+v^{L} \omega_{L}^{1}\right)+\omega^{3} \wedge \omega^{2} \wedge\left(d v^{2}+v^{L} \omega_{L}^{2}\right)+d \tau\left(v^{K} a_{K 2}^{1}-v^{K} a_{K 1}^{2}\right)\right) .
\end{aligned}
$$

Ввиду несжимаемости крови, ее объемный расход через замкнутую поверхность $S$ должен быть равен нулю. Поэтому

$$
\operatorname{div} \boldsymbol{v}=0 .
$$

С учетом (8), равенство (6) примет вид

$$
\begin{aligned}
\left(d v^{1}+v^{B} \omega_{B}^{1}\right) \wedge \omega^{2} \wedge \omega^{3}+\left(d v^{2}+v^{B} \omega_{B}^{2}\right) \wedge \omega^{3} \wedge \omega^{1}+ & \\
& +\left(d v^{3}+v^{B} \omega_{B}^{3}\right) \wedge \omega^{1} \wedge \omega^{2}+\left(v^{K} a_{K A}^{A}\right) \omega^{1} \wedge \omega^{2} \wedge \omega^{3}=0 .
\end{aligned}
$$

Выбирая вектор $\boldsymbol{e}_{3}$ по направлению касательной линии тока, перепишем соотношение (9) в виде

$$
v \omega_{3}^{1} \wedge \omega^{2} \wedge \omega^{3}+v \omega_{3}^{2} \wedge \omega^{3} \wedge \omega^{1} \wedge+(d v) \wedge \omega^{1} \wedge \omega^{2}+\left(\begin{array}{c}
v^{K} a_{K A}^{A} \\
K \neq A
\end{array}\right) \omega^{1} \wedge \omega^{2} \wedge \omega^{3}=0 .
$$

С учетом обозначений

$$
\omega_{3}^{1}=-\omega_{1}^{3}=q_{A} \omega^{A}=q ; \quad \omega_{2}^{3}=-\omega_{3}^{2}=p_{A} \omega^{A}=p,
$$

последнее уравнение принимает вид

$$
v q_{1} \omega^{1} \wedge \omega^{2} \wedge \omega^{3}-v p_{2} \omega^{2} \wedge \omega^{3} \wedge \omega^{1}+d v \wedge \omega^{1} \wedge \omega^{2}+v\left(a_{31}^{1}+a_{32}^{2}\right) \omega^{1} \wedge \omega^{2} \wedge \omega^{3}=0
$$

или

а также

$$
d v \wedge \omega^{1} \wedge \omega^{2}+v\left(q_{1}-p_{2}\right) \omega^{1} \wedge \omega^{2} \wedge \omega^{3}+v\left(a_{31}^{1}+a_{32}^{2}\right) \omega^{1} \wedge \omega^{2} \wedge \omega^{3}=0,
$$

$$
\frac{d v}{v} \wedge \omega^{1} \wedge \omega^{2}=\left(p_{2}-q_{1}\right) \omega^{1} \wedge \omega^{2} \wedge \omega^{3}-\left(a_{31}^{1}+a_{32}^{2}\right) \omega^{1} \wedge \omega^{2} \wedge \omega^{3} .
$$


Последнее равенство можно переписать следующим образом:

$$
\left(\frac{d \ln v}{d s}\right) \omega^{1} \wedge \omega^{2} \wedge \omega^{3}=\left(p_{2}-q_{1}-\left(a_{31}^{1}+a_{32}^{2}\right)\right) \omega^{1} \wedge \omega^{2} \wedge \omega^{3}
$$

или

$$
\frac{d \ln v}{d s}=p_{2}-q_{1}-\left(a_{31}^{1}+a_{32}^{2}\right) .
$$

Правая часть равенства (10) является средней кривизной векторного поля, или средней кривизной линий тока крови. Тем самым получена следующая теорема.

Теорема 1. В каждой точке потока крови логарифмическая производная от величины скорости по направлению линии тока равна средней кривизне конгруэнций линий тока крови.

Правая часть равенства (10) обращается в нуль тогда и только тогда, когда разность $p_{2}-q_{1}$ равна сумме первой и второй координат векторов второго порядка $\boldsymbol{e}_{31}$ и $\boldsymbol{e}_{32}$ соответственно. Конгруэнция линий тока в субпроективном пространстве, отнесенного к неголономному реперу, для которой $p_{2}-q_{2}-\left(a_{31}^{1}+a_{32}^{2}\right)=0$, назовем минимальной конгруэнцией. Тем самым доказана следующая теорема.

Теорема 2. Величина скорости потока крови в субпроективном пространстве, отнесенном $\kappa$ неголономным реперам, постоянна вдоль некоторой линии тогда и только тогда, когда данная линия принадлежит минимальной конгруэниии.

Пусть, как всегда, вихревой вектор имеет следующий вид:

$$
\boldsymbol{\nu}=\frac{1}{2} \operatorname{rot} \boldsymbol{v}=\frac{1}{2} \nu^{A} \boldsymbol{e}_{A} .
$$

Тогда из формулы (7) определим компоненты вихря.

Направим вектор $\boldsymbol{e}_{3}$ по касательной линии тока. Тогда последние формулы примут вид

$$
\begin{aligned}
& -\omega^{1} \wedge \omega^{2} \wedge \omega^{3} \times n^{1}=\omega^{1} \wedge \omega^{2} \wedge\left(v \omega_{3}^{2}\right)+\omega^{1} \wedge \omega^{3} \wedge d v+\omega^{1} \wedge \omega^{2} \wedge \omega^{3}\left(v a_{33}^{2}\right) ; \\
& -\omega^{1} \wedge \omega^{2} \wedge \omega^{3} \times n^{2}=\omega^{2} \wedge \omega^{1} \wedge\left(v \omega_{3}^{1}\right)+\omega^{2} \wedge \omega^{3} \wedge d v+\omega^{1} \wedge \omega^{2} \wedge \omega^{3}\left(-v a_{33}^{1}\right) ; \\
& -\omega^{1} \wedge \omega^{2} \wedge \omega^{3} \times n^{3}=\omega^{3} \wedge \omega^{1} \wedge\left(v \omega_{3}^{1}\right)+\omega^{3} \wedge \omega^{2} \wedge\left(v \omega_{3}^{2}\right)+\omega^{1} \wedge \omega^{2} \wedge \omega^{3}\left(v a_{32}^{1}-v a_{31}^{2}\right) .
\end{aligned}
$$

С учетом введенных выше обозначений для форм $\omega_{3}^{1}$ и $\omega_{1}^{3}$, а также $\omega_{2}^{3}$ и $\omega_{3}^{2}$ и $d v=v_{A} \omega^{A}$, запишем:

$$
\begin{aligned}
& -\omega^{1} \wedge \omega^{2} \wedge \omega^{3} \times n^{1}=\omega^{1} \wedge \omega^{2} \wedge\left(-v p_{3} \omega^{3}\right)+\omega^{1} \wedge \omega^{3} \wedge v_{2} \omega^{2}+\omega^{1} \wedge \omega^{2} \wedge \omega^{3}\left(v a_{33}^{2}\right) \\
& -\omega^{1} \wedge \omega^{2} \wedge \omega^{3} \times n^{2}=\omega^{2} \wedge \omega^{1} \wedge\left(v q_{3} \omega^{3}\right)+\omega^{2} \wedge \omega^{3} \wedge v_{1} \omega^{1}+\omega^{1} \wedge \omega^{2} \wedge \omega^{3}\left(-v a_{33}^{1}\right) ; \\
& -\omega^{1} \wedge \omega^{2} \wedge \omega^{3} \times n^{3}=\omega^{3} \wedge \omega^{1} \wedge\left(v q_{2} \omega^{2}\right)+\omega^{3} \wedge \omega^{2} \wedge\left(-v p_{1} \omega^{1}\right)+\omega^{1} \wedge \omega^{2} \wedge \omega^{3}\left(v a_{32}^{1}-v a_{31}^{2}\right) .
\end{aligned}
$$

Отсюда имеем

$$
-\nu^{1}=-v p_{3}-v_{2}+v a_{33}^{2} ; \quad-\nu^{2}=-v q_{3}+v_{1}-v a_{33}^{1} ; \quad-\nu^{3}=v q_{2}+v p_{1}+v a_{32}^{1}-v a_{31}^{2} .
$$

Из последней формулы системы (12) получим

$$
\frac{\nu^{3}}{v}=-\left(q_{2}+p_{1}+a_{32}^{1}-a_{31}^{2}\right)
$$

Гауссова кривизна векторного поля $\boldsymbol{e}_{3}$, коллинеарного вектору скорости, будет равна:

$$
\begin{aligned}
K_{g} & =-\left(p_{2}-\boldsymbol{e}_{32} \boldsymbol{e}_{2}\right)\left(q_{1}+\boldsymbol{e}_{31} \boldsymbol{e}_{1}\right)-\frac{1}{4}\left(p_{1}-q_{2}-\boldsymbol{e}_{32} \boldsymbol{e}_{1}-\boldsymbol{e}_{31} \boldsymbol{e}_{2}\right)^{2}= \\
& =-p_{2} q_{1}+q_{1} \boldsymbol{e}_{32} \boldsymbol{e}_{2}-p_{2} \boldsymbol{e}_{31} \boldsymbol{e}_{1}+\left(\boldsymbol{e}_{32} \boldsymbol{e}_{2}\right)\left(\boldsymbol{e}_{31} \boldsymbol{e}_{1}\right)-\frac{1}{4}\left(p_{1}-q_{2}-\boldsymbol{e}_{32} \boldsymbol{e}_{1}-\boldsymbol{e}_{31} \boldsymbol{e}_{2}\right)^{2}= \\
& =-p_{2} q_{1}+q_{1}\left(a_{32}^{1} \boldsymbol{e}_{1}+a_{32}^{2} \boldsymbol{e}_{2}\right) \boldsymbol{e}_{2}-p_{2}\left(a_{31}^{1} \boldsymbol{e}_{1}+a_{31}^{2} \boldsymbol{e}_{2}\right) \boldsymbol{e}_{1}+a_{32}^{2} a_{31}^{1}-\frac{1}{4}\left(p_{1}-q_{2}-a_{32}^{1}-a_{31}^{2}\right)^{2}= \\
& =-p_{2} q_{1}+q_{1} a_{32}^{2}-p_{2} a_{31}^{1}+a_{32}^{2} a_{31}^{1}-\frac{1}{4}\left(p_{1}-q_{2}-a_{32}^{1}-a_{31}^{2}\right)^{2} .
\end{aligned}
$$


Пусть $d_{1} \boldsymbol{x}$ и $d_{2} \boldsymbol{x}$ - два перемещения, ортогональных векторному полю $\boldsymbol{e}_{3}$. Рассмотрим отношение объемов параллелепипедов, построенных на тройке $\boldsymbol{e}_{3}, \boldsymbol{e}_{3}+d_{1} \boldsymbol{e}_{3}, \boldsymbol{e}_{3}+d_{2} \boldsymbol{e}_{3}$ и на тройке $\boldsymbol{e}_{3}, d_{1} \boldsymbol{x}, d_{2} \boldsymbol{x}$ в окрестности $U$ точки $x$ субпроективного пространства, отнесенного к неголономным реперам, и это отношение назовем полной кривизной поля $K_{t}$. На основании этого запишем:

$$
\begin{aligned}
& K_{t}=\frac{\boldsymbol{e}_{3}\left(\boldsymbol{e}_{3}+d_{1} \boldsymbol{e}_{3}\right) \wedge\left(\boldsymbol{e}_{3}+d_{2} \boldsymbol{e}_{3}\right)}{\boldsymbol{e}_{3} d_{1} \boldsymbol{x} \wedge d_{2} \boldsymbol{x}}=\frac{\boldsymbol{e}_{3}\left(\boldsymbol{e}_{3}+\left(q+\omega^{B} a_{3 B}^{1}\right) \boldsymbol{e}_{1}\right) \wedge\left(\boldsymbol{e}_{3}+\left(-p+\omega^{B} a_{3 B}^{2}\right) \boldsymbol{e}_{2}\right)}{\boldsymbol{e}_{3} d_{1} \boldsymbol{x} \wedge d_{2} \boldsymbol{x}}= \\
& +\frac{\left(p-\omega^{B} a_{3 B}^{2}\right) \wedge\left(q+\omega^{B} a_{3 B}^{1}\right)}{\omega^{1} \wedge \omega^{2}}= \\
& =\frac{\left(p_{1} \omega^{1}+p_{2} \omega^{2}-\omega^{1} a_{31}^{2}-\omega^{2} a_{32}^{2}\right) \wedge\left(q_{1} \omega^{1}+q_{2} \omega^{2}+\omega^{1} a_{31}^{1}+\omega^{2} a_{32}^{1}\right)}{\omega^{1} \wedge \omega^{2}}= \\
& =\frac{p_{1} q_{2} \omega^{1} \wedge \omega^{2}+p_{1} a_{32}^{1} \omega^{1} \wedge \omega^{2}-p_{2} q_{1} \omega^{1} \wedge \omega^{2}-p_{2} a_{31}^{1} \omega^{1} \wedge \omega^{2}-q_{2} a_{31}^{2} \omega^{1} \wedge \omega^{2}-}{\omega^{1} \wedge \omega^{2}}+ \\
& +\frac{-a_{31}^{2} a_{32}^{1} \omega^{1} \wedge \omega^{2}+q_{1} a_{32}^{2} \omega^{1} \wedge \omega^{2}+a_{31}^{1} a_{32}^{2} \omega^{1} \wedge \omega^{2}}{\omega^{1} \wedge \omega^{2}}= \\
& =p_{1} q_{2}+p_{1} a_{32}^{1}-p_{2} q_{1}-p_{2} a_{31}^{1}-q_{2} a_{31}^{2}-a_{31}^{2} a_{32}^{1}+q_{1} a_{32}^{2}+a_{31}^{1} a_{32}^{2} \text {. }
\end{aligned}
$$

Тогда

$$
K_{t}-K_{g}=p_{1} q_{2}+p_{1} a_{32}^{1}-q_{2} a_{31}^{2}-a_{31}^{2} a_{32}^{1}+\frac{1}{4}\left(p_{1}-q_{2}-a_{32}^{1}-a_{31}^{2}\right)^{2}=\frac{1}{4}\left(p_{1}+q_{2}-\left(a_{31}^{2}-a_{32}^{1}\right)\right)^{2}
$$

Итак, имеем

$$
\sqrt{K_{t}-K_{g}}=\frac{1}{2}\left|p_{1}+q_{2}+a_{32}^{1}-a_{31}^{2}\right|
$$

Сравнивая последнюю формулу с (13), получим

$$
\frac{\left|\nu^{3}\right|}{v}=\left|q_{2}+p_{1}+a_{32}^{1}-a_{31}^{2}\right|=2 \sqrt{K_{t}-K_{g}}
$$

Теорема 3. Отношение проекции вихря на касательную линии тока крови к величине скорости крови есть инвариант линии тока, который пропорционален квадратному корню из разности полной и гауссовой кривизны линии тока крови.

\section{СПИСОК ЛИТЕРАТУРЫ}

1. Кузнецов Г. В. Геометрические объекты и параметры сердечно-сосудистой системы. - Тула: Промпилот, 2017.

Кузнецов Геннадий Васильевич

Финансовый университет при Правительстве Российской Федерации, Тульский филиал E-mail: tula@fa.ru 